\section{INJURY ALLIANCE IN CANADA}

P Fuselli*, P Kellsbert Baker, R Nesdale-Tucker Correspondence: Safe Kids Canada/ The Hospital for Sick Children, 180 Dundas Street West Suite 2105, Toronto, Ontario, M5G 1Z8, Canada

10.1136/ip.2010.029215.381

To achieve greater societal impact within a highly competitive charitable marketplace, the four major, national, injury prevention organisations have decided to collaborate toward the following purposes:

Long-term goal To save more lives and prevent more potentially life-altering injuries in Canada.

Medium-term objective To raise awareness and to facilitate attitudinal and behavioural shifts among the organisations respective target populations, in partnership with their natural allies, of best practices in preventing injuries, and injuryrelated deaths in Canada.

Short-term output The development of recommendations, an implementation plan and supporting documentation for a game changer that will build their capacity as individual organisations to promote more effective and efficient strategies and programming.

Seek better ways and means to identify, integrate and approve the collaborative delivery of:

- knowledge creation, synthesis and transfer

- stakeholder engagement

- fund development

marketing.

This presentation will share the outcomes of phase I of the study in terms of defining the ideal inputs and outputs, catalogue these inputs and outputs in relation to the sponsoring organisations, describe what is unique about those inputs and outputs among the sponsoring organisations and what is shared by two or more of them, project and outline the benefits that the collaborative delivery of the function could bring, advance recommendations for amending existing processes, or introduce new ones for more suitably identifying, overseeing, managing and assessing future shared initiatives within each function, describe one or more approaches for resourcing such collaborative processes on a go-forward basis, propose a strategic or 3-year outlook for the frameworks operation. 